\title{
A STUDY OF MAIN SEQUENCE EARLY TYPE STARS
}

\author{
Uf Sinnerstad
}

Stockholn Observatory

ABSTRACT

A spectroscopic and photometric study of 75 normal main sequence B2-B6 stars has been carried out. From the spectra (12 $\AA / \mathrm{mm}, 3700-4800$ \&) are determined: equivalent widths of all measurable lines, line profiles of hydrogen lines and $\mathrm{v}$ sin $i$. The photometric work includes besides uvby and $\beta$ photometry, also photoelectrically determined line strengths of $\mathrm{H}_{\gamma}$ and $\mathrm{He} I 4471$ A. Some preliminary results concerning the relation between the MK types and the atmospheric fundamental parameters $\left(\mathrm{T}_{\mathrm{eff}}, \log \mathrm{g}\right)$ of the stars are briefly discussed.

Three methods are available for estimating the physical properties of a star: spectrophotometry, direct photoelectric photometry and classification by visual inspection of the spectrum. It is well known that a two-dimensional classification scheme is sufficient for classifying all normal early type stars and that these stars can be characterized adequately by two photometric indices. The reason is the also well known fact that two atmospheric parameters - the effective temperature $T_{e f f}$ and the surface gravity 8 - dominate over all other parameters such as difference in chemical composition, axial rotation, turbulent velocity and magnetic field. The predominant influence of the two parameters also implies that the stellar spectra are highly redundant: the appearance of different spectral lines or regions are strongly correlated to each other as the spectral features mainly carry the same information.

The presence of other parameters and the complexity of the physics involved in the stellar atmosphere give good reasons, however, to suspect that there can be no unique relation between for 
example the photoelectric indices in the uvby and system on the one hand and the $M \mathbb{K}$ classes on the other hand. Neither is it to be expected that spectral lines formed in different parts of the stellar atmosphere such as strong lines and faint lines will show a one to one correspondence to the photoelectric indices or the MK type.

The aim of the present work is to look into these problems for a rather restricted and homogeneous group of stars, the main sequence B2-B6 stars which are of great importance both from the point of view of the study of stellar age and distance. Normal B2-B6 stars in the Bright Star Catalogue with $V \leq 6^{m}$ not classified as supergiants were selected for the investigation. Stars marked as variables, stars with companions closer than $\mathrm{d}=15^{\prime \prime}$ and/or $\Delta \mathrm{m}$ $\leq 4 \mathrm{~m}$, and stars with variable radial velocity were excluded. The Be stars are included as the $e$ - characteristics have not been considered as abnormal.

The resulting 75 stars within the coordinate limits $6 \mathrm{~h} \leq \alpha \leq 18^{\mathrm{h}}$, $\delta<-10^{\circ}$ were observed at ESO, Ia Silla, in February and March, 1972 with the coude spectrograph of the $152 \mathrm{~cm}$ telescope and with the Roden photometer on the ESO $50 \mathrm{~cm}$ telescope. The spectrographic material which will be discussed in Astronomy and Astrophysics consists of two $12 \AA / \mathrm{mm}$ spectra of each star in the wavelength region 3700-4800 $\AA$ and has been used to determine the equivalent widths of all measurable lines and line profiles of strong lines.

The photometric observations consist of Stromgren four color and $\beta$ indices and indices for the line strengths of $\mathrm{H} \gamma$ and $\mathrm{He} 4471$. Almost all program stars already had well determined uvby and $\rho$ indices but since more than $20 \%$ of the program stars are $\mathrm{Be}$ or suspected Be stars new measurements were made for all stars in the program for checking variability. The reductions were made as recommended by Crawford and Barnes (1970) and Crawford and Mander (1965). The corrections for interstellar reddening were determined using the relations given by Crawford (1975). Comparison with stars in common with Crawford et al. (1970, 1971) omitting all Be stars shows that the mean errors are \pm .009 in $\beta, \pm .007$ in $u-b$, \pm .011 in $b-y$ and \pm .011 in $c_{1}$. Furthe $\bar{r}$ details will be published in Astronomy and Astrophysics.

One specific problem will be touched upon in the following: the relation between the $\mathbb{M K}$ classes and the fundamental parameters Teff and g. Data for the program stars - the Be stars and a few peculiar stars omitted - are given in Table I where the MK classes are from Hiltner et al. (1969) or Lesh (1968), and data for $M$ standard stars is contained in Table II. The parameters $\theta_{\text {eff }}=$ 
TABLE I

PROGRAY STARS

\begin{tabular}{|c|c|c|c|c|c|c|}
\hline \multirow{3}{*}{$\begin{array}{l}\mathrm{HD} \\
41814 \\
42927\end{array}$} & \multicolumn{2}{|c|}{$\begin{array}{c}\text { Spectral } \\
\text { Type }\end{array}$} & \multirow{3}{*}{$\frac{(u-b)}{0}$} & \multirow{3}{*}{$\begin{array}{c}B \\
2.682 \\
2.664\end{array}$} & \multirow{3}{*}{$\begin{array}{r}\theta_{\text {eff }} \\
0.289 \\
.283\end{array}$} & \multirow{2}{*}{$\frac{\log 8}{4.09}$} \\
\hline & B3 & $\mathbf{v}$ & & & & \\
\hline & B3 & III & & & & 3.97 \\
\hline 51283 & B2 & III & .130 & 2.589 & .257 & 3.39 \\
\hline 52092 & B3 & $\mathbf{v}$ & .385 & 2.680 & .311 & 3.89 \\
\hline 56342 & B3 & $\mathbf{v}$ & .387 & 2.679 & .312 & 3.88 \\
\hline 57219 & B2 & IV & .224 & 2.666 & .268 & 4.12 \\
\hline 58286 & B2 & $\mathbf{v}$ & .311 & 2.649 & .294 & 3.73 \\
\hline 61068 & B2 & III & -.004 & 2.615 & .213 & 4.10 \\
\hline 61831 & B2. 5 & $\mathbf{V}$ & .333 & 2.670 & .298 & 3.90 \\
\hline 63465 & B2. 5 & III & .303 & 2.636 & .293 & 3.61 \\
\hline 64740 & B1. 5 & $V_{p}$ & -.018 & 2.622 & .209 & 4.23 \\
\hline 64802 & B2 & $\mathbf{v}$ & .250 & 2.658 & .276 & 3.97 \\
\hline 65575 & B3 & $I V_{p}$ & .351 & 2.659 & .304 & 3.75 \\
\hline 65904 & B4 & V & .386 & 2.682 & .311 & 3.91 \\
\hline 66591 & B3 & v & .354 & 2.676 & .303 & 3.92 \\
\hline 68217 & B2 & IV-V & .228 & 2.638 & .272 & 3.80 \\
\hline 69081 & $\mathrm{Bl} .5$ & IV & .032 & 2.617 & .221 & 4.04 \\
\hline 71459 & B3 & $\mathbf{v}$ & .409 & 2.674 & .318 & 3.78 \\
\hline 71510 & B2 & $\mathbf{V}$ & .307 & 2.666 & .291 & 3.92 \\
\hline 74071 & BS & v & .487 & 2.705 & .336 & 3.93 \\
\hline 75575 & B1. 5 & III & -.024 & 2.603 & .209 & 3.99 \\
\hline 76538 & B5 & III & .448 & 2.649 & .333 & 3.44 \\
\hline 77002 & B2 & IV $-\mathrm{V}$ & .223 & 2.658 & .269 & 4.03 \\
\hline 79447 & B3 & III & .321 & 2.658 & .296 & 3.80 \\
\hline 79735 & 84 & $\mathbf{v}$ & .463 & 2.677 & .332 & 3.71 \\
\hline 86406 & B3 & IV & .409 & 2.648 & .321 & 3.52 \\
\hline 92938 & B4 & IV & .444 & 2.697 & .325 & 3.94 \\
\hline 93194 & B4 & IVn & .406 & 2.665 & .318 & 3.70 \\
\hline 93607 & B3 & v & .321 & 2.674 & .294 & 3.97 \\
\hline 93845 & B2. 5 & IV & .270 & 2.677 & .280 & 4.12 \\
\hline 98695 & $B 4$ & v & .391 & 2.692 & .311 & 4.00 \\
\hline 99556 & 83 & IV & .409 & 2.634 & .324 & 3.36 \\
\hline 100929 & B2. 5 & IV & .326 & 2.637 & .300 & 3.56 \\
\hline 106490 & B2 & IV & -.001 & 2.626 & .212 & 4.24 \\
\hline 106911 & B5 & $V_{n}$ & .525 & 2.704 & .346 & 3.85 \\
\hline 106983 & B2. 5 & $\mathbf{v}$ & .270 & 2.679 & .279 & 4.15 \\
\hline 108483 & B2 & $\mathbf{V}$ & .131 & 2.659 & .243 & 4.29 \\
\hline 112078 & 84 & $V_{n}$ & .395 & 2.679 & .313 & 3.87 \\
\hline 112092 & B2 & $I V-V$ & .167 & 2.655 & .253 & 4.15 \\
\hline 113703 & $B 5$ & $\mathbf{v}$ & .432 & 2.708 & .321 & 4.07 \\
\hline 120908 & B5 & III & .555 & 2.680 & .356 & 3.57 \\
\hline 122980 & $B 2$ & $\mathbf{v}$ & .158 & 2.657 & .251 & 4.19 \\
\hline 125238 & 82.5 & IV & .246 & 2.651 & .275 & 3.90 \\
\hline 126341 & B2 & IV & .070 & 2.620 & .232 & 3.96 \\
\hline 128345 & 85 & $\mathbf{v}$ & .437 & 2.705 & .322 & 4.04 \\
\hline 132955 & B3 & $\mathbf{v}$ & .373 & 2.703 & .305 & 4.16 \\
\hline 136298 & 81.5 & IV & .013 & 2.616 & .216 & 4.08 \\
\hline 136664 & $B 4$ & $\mathbf{v}$ & .345 & 2.690 & .298 & 4.09 \\
\hline 142184 & B2. 5 & $v$ & .293 & 2.657 & .289 & 3.85 \\
\hline 142669 & B2 & $I V-V$ & .102 & 2.642 & .237 & 4.16 \\
\hline 142883 & B3 & $\mathbf{V}$ & .425 & 2.713 & .320 & 4.12 \\
\hline 142919 & BS & IV & .486 & 2.643 & .344 & 3.31 \\
\hline 144294 & B2. 5 & Vn & .237 & 2.661 & .271 & 4.04 \\
\hline 150745 & B2 & $I V-V$ & .243 & 2.630 & .278 & 3.66 \\
\hline
\end{tabular}


$\frac{5040}{T}$ and $\log \mathrm{g}$ have been derived from the uvby and $\beta$ photometry as follows :

1.) The relation

$$
\theta_{\text {eff }}=0.218+0.227(u-b)_{0}
$$

was derived from the six stars (Table III) within the spectral class range B1-B7 with empirically determined effective temperatures by Code et al. (1976) and with uvby indices in the catalogue by Lindemann and Hauck (1973).

2.) From the measurements of the equivalent widths of $H_{\gamma}$ and $\mathrm{H}_{\delta}$ and from the indices of the program stars the relation

$$
W_{\nexists \delta}(20)=-73.5+29.9 p
$$

was found, where $\mathrm{W}_{\gamma \delta}(20)$ means the arithmetic mean value of the equivalent widths of $\mathrm{H}_{\gamma}$ and $\mathrm{H}_{\delta}$ within the wavelength limits +20 \& from the line centers. The same quantity, $W_{\delta \delta}(20)$, was also computed from the grid of models given by Kurucz, Peytremann and Avrett (1974) - in the following called the KPA model - and used with 0 ef from relation (I) for obtaining log $g$.

3.) These $\log g$ values were then used with $(u-b)_{0}$ to obtain

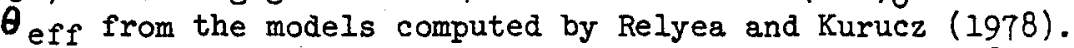
The new values are slightly dependent on the gravity [an approximate relation for the spectral classes in question is $\theta_{\text {eff }}=0.213+0.250(u-b)_{0}-0.014(10 g$ g-4.00)] but still in close agreement with the empirically derived effective temperatures as seen in Table III.

4.) Finally the new $\theta_{\text {eff }}$ values were used to derive the tabulated $\log \mathrm{g}$ from the KPA model. The final $\log _{\mathrm{g}}$ values differ from those first derived by less than 0.10 .

In order to estimate the intemal accuracy of the parameters just obtained the mean error in each of the indices $(u-b),(b-y)$ and $\beta$ has been assumed to be \pm 0 m010. The corresponding mean error in $\theta_{\text {eff }}$ is \pm .007 and in $\log 6 \pm .12$.

As the importance of the line wings has been suppressed in the present method in calculating the surface gravity, the $\log g$ values have been ehecked for the program stars by measuring also the $\mathrm{H}_{\mathcal{S}}$ and $\mathrm{H}_{\gamma}$ line widths at $80 \%$ and $90 \%$ of the continuum intensity and comparing the widths with the theoretical profiles in the KPA 
model. The mean difference, $\Delta \log \mathrm{B}$, between the two methods is $0.04 \pm 0.17$ as computed for all program stars in Table $I$. No significant systematic effects in $\Delta \log g$ could be detected for different $\log g$ or $\theta$ eff.

The standard stars in Table II are plotted in a $\theta_{\text {eff, }}$ log $g$ diagram (Fig. 1). Large symbols indicate the primary standards in the MK system, the smaller ones other standards used by Lesh (1968) and by Hiltner et al. (1969). The straight lines separating spectral and luminosity classes are eye estimates of the best fit to the symbols with special regard to the primary standards. The lines separating the spectral classes are rather uncertain, of course, on account of the few stars in each class. The dashed lines connect points derived from the uvby and $\beta$ indices given by Crawford (1978) for the zero age main sequence and from the average indices given by him for the MK types considered.

Some interesting points derived from the MK standard stars and from Crawford's average indices will be mentioned here.

1. There is a very good agreement between the MK classes and the values of $\boldsymbol{\theta}_{\text {eff }}$ and $\log g$ for the primary standards.

2. The agreement is also good for the other standards, with a few exceptions such as HD 32612 and HD 197511.

3. The average parameters for luminosity class $V$ fall mainly in the region for luminosity class IV of the standard stars.

4. The average parameters for luminosity class III are generally displaced from the class III standards towards the region for luminosity class IV of the standard stars.

The difference between the $\log \mathrm{g}$ values for the average $M K$ luminosity classes III and $V$ is thus smaller than the corresponding difference for the $M K$ standard stars. This is also apparent in the case of the program stars. The difference between the $10 \mathrm{~g} g$ values for the program stars of luminosity classes III and $V$ is 0.25 on an average while the corresponding mean difference for the $M K$ standard stars is 0.50 .

A reclassification of the program stars according to Fig. 1 results in changes of luminosity and spectral classes as shown in Table IV, where positive $\Delta S$ means a change to a later spectral class and positive $\Delta L$ to a higher luminosity class. As seen from Table IV there is a strong correlation between changes in spectral and Iuminosity classes in such a way that a change to a lower 
TABLE II

STANDARD STARS

(Spectral types underlined are primary standards)

\begin{tabular}{|c|c|c|c|c|c|c|}
\hline HD & $\begin{array}{c}\text { Spect } \\
\text { Tyo }\end{array}$ & $\begin{array}{l}8 \mathrm{l} \\
\mathrm{e}\end{array}$ & $(u-b)_{0}$ & 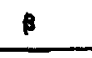 & eff & $\log 8$ \\
\hline $\begin{array}{r}886 \\
3360 \\
4727 \\
16582 \\
22928 \\
23302 \\
23338 \\
30836 \\
32612 \\
32630 \\
34503 \\
34759 \\
35468 \\
36779 \\
37711 \\
90994 \\
120315 \\
147394 \\
148605 \\
175191 \\
176502 \\
178329 \\
197511 \\
207330 \\
215191 \\
216200\end{array}$ & $\begin{array}{l}\text { B2 } \\
B 2 \\
B 5 \\
B 2 \\
B 5 \\
B 6 \\
B 6 \\
B 2 \\
B 2.5 \\
B 3 \\
B 5 \\
B 5 \\
B 2 \\
B 2.5 \\
B 3 \\
B 6 \\
B 3 \\
B 5 \\
B 2 \\
B 2.5 \\
B 4 \\
B 3 \\
B 2 \\
B 2.5 \\
B 1.5 \\
B 4\end{array}$ & $\begin{array}{l}I V \\
I V \\
V V \\
I V \\
I I I \\
I I I \\
I V \\
I I I \\
I V \\
V \\
I I I \\
V \\
I I I \\
V \\
I V \\
V \\
V \\
I V \\
V \\
V V \\
I V \\
V \\
V \\
I I I \\
V \\
I I I\end{array}$ & $\begin{array}{r}0.092 \\
.106 \\
.478 \\
.060 \\
.574 \\
.727 \\
.638 \\
.081 \\
.181 \\
.355 \\
.680 \\
.471 \\
.057 \\
.112 \\
.327 \\
.558 \\
.336 \\
.447 \\
.197 \\
.221 \\
.337 \\
.356 \\
.300 \\
.232 \\
.038 \\
.321\end{array}$ & $\begin{array}{l}2.629 \\
2.625 \\
2.710 \\
2.624 \\
2.671 \\
2.690 \\
2.703 \\
2.606 \\
2.665 \\
2.684 \\
2.687 \\
2.722 \\
2.614 \\
2.644 \\
2.672 \\
2.730 \\
2.694 \\
2.702 \\
2.662 \\
2.667 \\
2.667 \\
2.690 \\
2.651 \\
2.617 \\
2.633 \\
2.634\end{array}$ & $\begin{array}{l}0.235 \\
.239 \\
.331 \\
.227 \\
.359 \\
.395 \\
.372 \\
.236 \\
.254 \\
.300 \\
.385 \\
.329 \\
.227 \\
.238 \\
.293 \\
.350 \\
.293 \\
.330 \\
.259 \\
.265 \\
.296 \\
.300 \\
.288 \\
.275 \\
.221 \\
.298\end{array}$ & $\begin{array}{l}4.04 \\
3.95 \\
4.01 \\
4.06 \\
3.47 \\
3.40 \\
3.66 \\
3.76 \\
4.24 \\
4.02 \\
3.44 \\
4.13 \\
3.94 \\
4.17 \\
3.96 \\
4.04 \\
4.18 \\
3.95 \\
4.16 \\
4.16 \\
3.89 \\
4.08 \\
3.80 \\
3.55 \\
4.23 \\
3.55\end{array}$ \\
\hline
\end{tabular}

TABLE III

STARS WITH EMPIRICALLY DETERMINED EFFECTIVE TEMPERATURES

\begin{tabular}{|c|c|c|c|c|c|c|c|}
\hline HD & $\begin{array}{r}\text { Spe } \\
T\end{array}$ & $\begin{array}{l}\text { ctral } \\
\text { ype }\end{array}$ & $(u-b)_{0}$ & B & $\epsilon_{\text {eff }}$ & $\theta_{\text {enp }}$ & \\
\hline $\begin{array}{r}35468 \\
87901 \\
116658 \\
118716 \\
193294 \\
209952\end{array}$ & $\begin{array}{l}\text { B2 } \\
\text { B7 } \\
\text { B1 } \\
\text { B2 } \\
B 2.5 \\
B 7\end{array}$ & $\begin{array}{l}\text { III } \\
V \\
V \\
\text { III } \\
V \\
\text { IV }\end{array}$ & $\begin{array}{r}0.057 \\
.824 \\
-.051 \\
-.059 \\
.264 \\
.065\end{array}$ & $\begin{array}{l}2.614 \\
2.723 \\
2.607 \\
2.610 \\
2.660 \\
2.727\end{array}$ & $\begin{array}{r}0.229 \\
.411 \\
.203 \\
.199 \\
.277 \\
.375\end{array}$ & $\begin{array}{r}0.234 \\
.413 \\
.211 \\
.196 \\
.282 \\
.359\end{array}$ & $\begin{array}{r} \pm .009 \\
.010 \\
.007 \\
.009 \\
.011 \\
.014\end{array}$ \\
\hline
\end{tabular}

TABLE IV

CHANGES IN SPECTRAL CLASSIFICATION FOR PROGPAM STARS

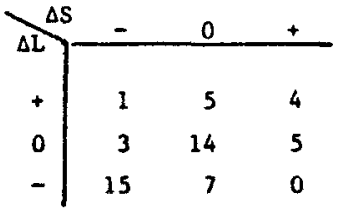




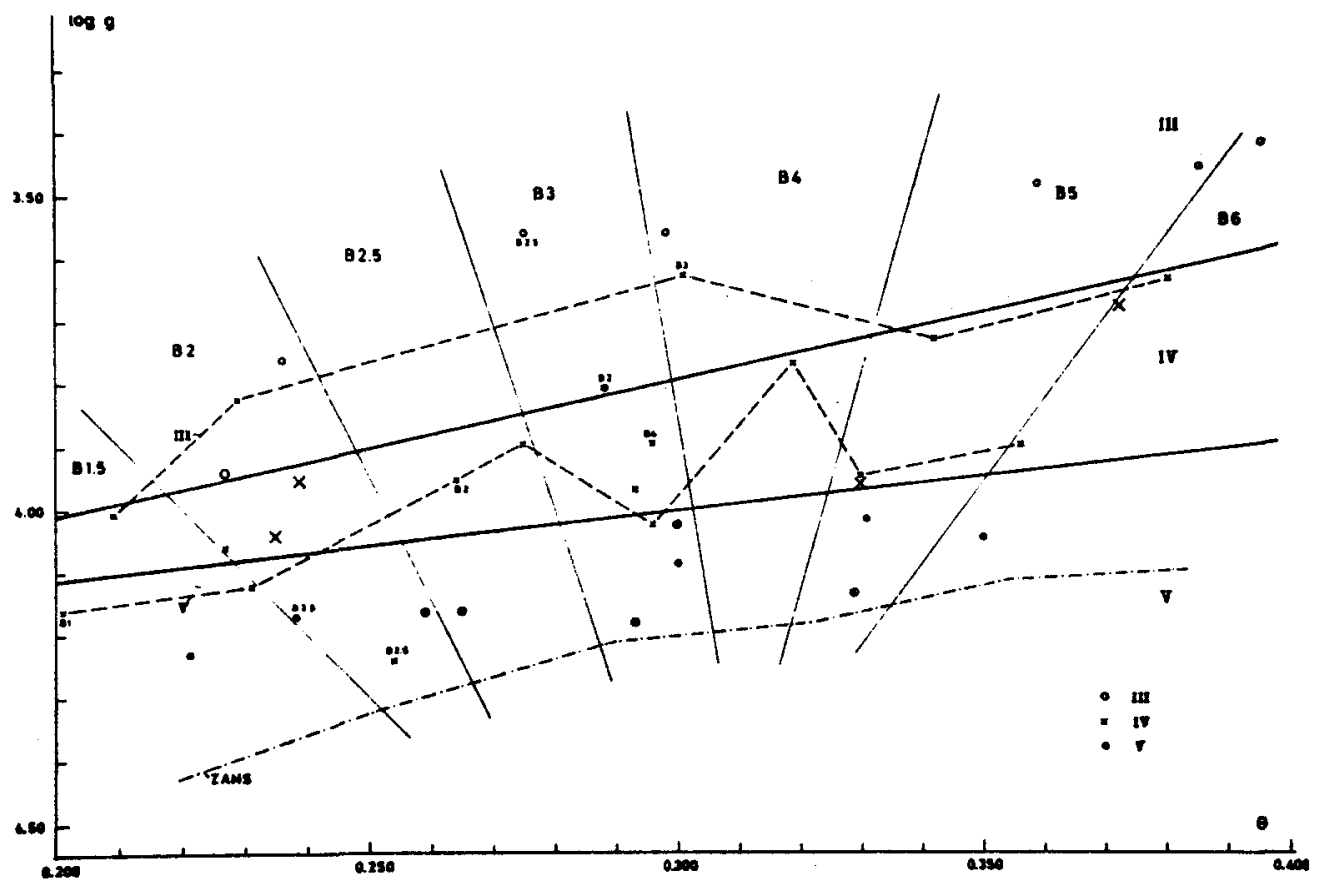

Fig. 1. A $\theta_{\text {eff }}$ vs log $g$ diagram for MK standard stars. Different symbols are used for the luminosity classes. Primary $M$ standards are marked with large symbols. Spectral types not in accordance with the empirically drawn grid are indicated. The broken lines connect the average positions (marked as crosses with the spectral class if deviating from the grid) for Iuminosity class III and V. They are based on the four color and $\beta$ indices given by Crawford (1978). The dashed dot line representing the zero age main sequence (ZAMS) is also derived from Crawford's data (1978). 
Iuminosity class is generally coupled to a change to an earlier spectral type. The changes are thus in the direction of constant hydrogen line intensity.

This work is supported by a grant from the Swedish Natural Science Research Council.

REFERENCES

Code, A.D., Davis, J., Bless, R.L. and Hanbury Brown, R. (1976). Astrophys. J. 203, 417.

Crawford, D.I. (1975). Publ. Astron. Soc. Pacific 87, 481.

Crawford, D. L. (1978). Astron. J. 83, 48.

Crawford, D.I. and Barnes, J.V. (1970). Astron. J. 75, 978.

Crawford, D.L., Barnes, J.V. and Golson, J.C. (1970). Astron. J. 75,624 .

Crawford, D.L., Barnes, J.V. and Golson, J.C. (1971). Astron. J. 76,621 .

Crawford, D.L. and Mander, J. (1965). Astron. J. 71, 114.

Hiltner, W.A., Garrison, R.F. and Schild, R.E. (1969). Astrophys. J. $157,313$.

Kurucz, R.I., Peytremann, E. and Avrett, E.H. (1974). In Blanketed Model Atmospheres for Early-Type Stars, Smithsonian Insitution: Washington, D.C.

Lesh, J.R. (1968). Astrophys. J. Suppl. 17, 371.

Iindemann, E. and Hauck, B. (1973). Astron. Astrophys. Suppl. 11, 119 .

Relyea, L.J. and Kurucz, R.I. (1978). Astrophys. J. Suppl. 37, 45. 